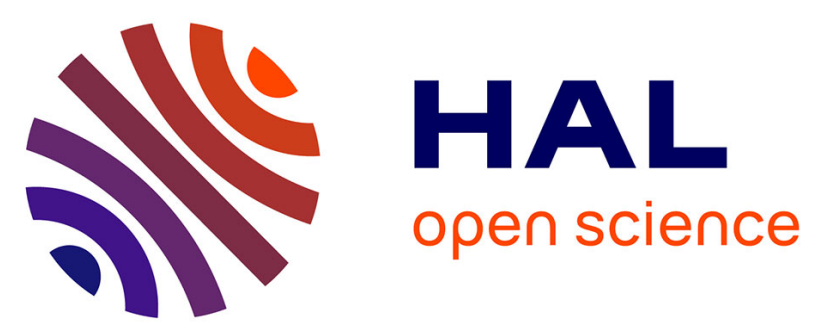

\title{
Assessment of Cirrus Cloud Optical and Microphysical Data Reliability by Applying Statistical Procedures
}

Valery Shcherbakov, Jean-François Gayet, Olivier Jourdan, Andreas Minikin, Johan Ström, Andreas Petzold

\section{To cite this version:}

Valery Shcherbakov, Jean-François Gayet, Olivier Jourdan, Andreas Minikin, Johan Ström, et al.. Assessment of Cirrus Cloud Optical and Microphysical Data Reliability by Applying Statistical Procedures. Journal of Atmospheric and Oceanic Technology, 2005, 22 (4), pp.409 - 420. 10.1175/JTECH1710.1 . hal-01823433

\section{HAL Id: hal-01823433 \\ https://hal.uca.fr/hal-01823433}

Submitted on 20 Oct 2021

HAL is a multi-disciplinary open access archive for the deposit and dissemination of scientific research documents, whether they are published or not. The documents may come from teaching and research institutions in France or abroad, or from public or private research centers.
L'archive ouverte pluridisciplinaire HAL, est destinée au dépôt et à la diffusion de documents scientifiques de niveau recherche, publiés ou non, émanant des établissements d'enseignement et de recherche français ou étrangers, des laboratoires publics ou privés. 


\title{
Assessment of Cirrus Cloud Optical and Microphysical Data Reliability by Applying Statistical Procedures
}

\author{
VALERY SHCHERBAKOV* \\ Institute of Physics, Minsk, Belarus \\ Jean-François Gayet and Olivier Jourdan \\ Laboratoire de Météorologie Physique, UMR/CNRS, Université Blaise Pascal, Clermont-Ferrand, France \\ ANDREAS MINIKIN \\ Institut für Physik der Atmosphäre, DLR, Oberpfaffenhofen, Germany \\ JOHAN STRÖM \\ Institute of Applied Environmental Research, Stockholm University, Stockholm, Sweden \\ Andreas Petzold \\ Institut für Physik der Atmosphäre, DLR, Oberpfaffenhofen, Germany
}

(Manuscript received 6 May 2004, in final form 15 September 2004)

\begin{abstract}
A methodology of employing statistical procedures, specifically the principal component analysis (PCA) technique, to assess cirrus cloud data reliability is described. The approach is demonstrated by an example of a study of optical and microphysical characteristics measured during two campaigns performed at midlatitudes in the pristine Southern ( $\mathrm{SH}$ ) and polluted Northern (NH) Hemispheres within the international INCA project (Interhemispheric Differences in Cirrus Cloud Properties from Anthropogenic Emissions). The datasets were obtained by using state-of-the-art airborne instruments including the polar nephelometer and PMS particle size spectrometers for the ice-particle characterization. The approach is applied to both the measured angular scattering intensities and the ice-particle size distributions. It is shown that the PCA technique allows for impartial elimination of nonreliable channels of instruments. Furthermore, this technique is efficient in a study if the dataset is statistically homogeneous, and provides the possibility of removing specific records corresponding to distinguishing statistical ensembles. The results, expressed in terms of significant components and corresponding eigenvalues, show that the Southern and Northern Hemisphere datasets are in good agreement and they can be considered as statistically representative of the sampled cirrus. Furthermore, the frequency distributions of the cirrus cloud microphysical and optical properties can be regarded as arbitrary positive quantities, which are lognormally distributed. The validation of the measurements is provided by intercomparison of parameters estimated from different and independent techniques. The statistical relationships between quantities derived from angular scattering intensities and from ice-particle distributions as well as the similarity of the results obtained for the Southern and Northern Hemisphere cases serve as proof of the reliability of the measured cloud properties.
\end{abstract}

\footnotetext{
* Additional affilation: OPGC, Laboratoire de Météorologie Physique, UMR/CNRS, Université Blaise Pascal, ClermontFerrand, France.
}

Corresponding author address: Dr. J.-F. Gayet, Laboratoire de Météorologie Physique, Université Blaise Pascal, 24, Avenue des Landais, 63177 Aubière Cedex, France.

E-mail: J.F.Gayet@opgc.univ-bpclermont.fr

\section{Introduction}

It is generally recognized that inadequate parameterization of ice-phase clouds is one of the greatest sources of uncertainty in both the modeling of climate processes and the interpretation of satellite remote sensing data (see, among others, Gonzalez et al. 2002). Accurate determination of their optical and microphysical 
characteristics is also crucial for improved understanding of cloud formation and evolution (see, among others, Platt et al. 1989). For this reason, several intensive field campaigns have been carried out over the last two decades in order to improve our knowledge of icephase cloud systems-for example, the First International Satellite Cloud Climatology Project (ISCCP) Regional Experiment (FIRE) experiments (Starr and Wylie 1990; Randall et al. 1995), the International Cirrus Experiment (ICE'89; Raschke et al. 1990), the European Cloud and Radiation Experiment (EUCREX'94; Sauvage et al. 1999), Formation Processes and Radiative Properties of Particles in Aircraft Wakes (AEROCONTRAIL; Schröder et al. 2000), and the Subsonic Aircraft: Contrail and Cloud Effects Special Study (SUCCESS; Lawson et al. 1998). Usually these experiments involve airborne instruments that simultaneously measure microphysical, optical, and other characteristics of clouds as well as meteorological parameters. Due to the well-known instrument shortcomings in the presence of small ice crystals in cirrus clouds (Gayet et al. 2002b) and because of the diversity of shapes and internal structures of ice particles (see, e.g., Heymsfield et al. 2002), the measurements must be carefully analyzed in order to reduce the uncertainties on the derived parameters.

Nowadays, a great deal of attention is paid to data reliability in the atmospheric sciences. Conferences have even featured special sessions devoted to the problem. As an example, we note the "Data Reliability and Usability" session at the 13th Conference on Applied Climatology of the American Meteorological Society (AMS 2002). We have not succeeded in finding out a strict definition of the term "data reliability" in the context of earth sciences. For social research methods, McNeil (1990) defined data reliability in the following terms: "If a method of collecting evidence is reliable it means that anybody using this method, or the same person using it at another time, would come up with the same results. The work could be repeated and the same results gained." And, we believe that McNeil's definition can serve for the atmospheric sciences. Of course, this definition should be considered along with the fact that instruments are dedicated to measure the physical quantities and, in principle, it may be possible to ensure data reliability by high-precision probes.

As far as measurement in cirrus clouds is concerned, the data reliability is usually ensured by rigorous calibrations and repeated checks of the instruments before and during campaigns. An outstanding example of thorough calibrations is the laboratory measurements of the response of a PMS OAP-2DC performed by Strapp et al. (2001). Another widely used approach consists in validation-that is, comparing the same parameter measured by different instruments using independent techniques. The case when the same parameter is estimated from data recorded by different probes (see, e.g., Gayet et al. 2002b) directly relates to this methodology.

From our standpoint, calibration and validations are necessary, however, not entirely sufficient for data reliability assessment. Nowadays, datasets of routine or campaign measurements are usually too huge to be manually checked by an operator through all records. For instance, both approaches mentioned above are well established in meteorology. In addition, statistical methods are employed in meteorological data qualitycontrol processes (Menne and Duchon 2001) despite the fact that, for example, temperature measurements are performed correctly. Statistical procedures provide checks for errors like electronic noise, defective records, a scale shift of the instrument, etc.

Compared to meteorological quantities, the measurement of cirrus cloud optical and microphysical parameters is a much more challenging task. Consequently, we believe that not only do calibrations and validations have to be employed in the control of the reliability of the measured and estimated data, but methods of statistics as well. Statistical procedures can serve as powerful tools providing additional, but necessary, checks. With McNeil's definition in mind, the following tactics of data reliability assessment can be employed. If several datasets are obtained in similar conditions and with clouds having the same properties, the most fundamental statistical features have to be shared by the datasets. Furthermore, the difference comes out in parameters like mean, variance, etc. These parameters serve as degrees of freedom. If a dataset (or several datasets) significantly differs by its main features from a whole ensemble, at least it means that special attention has to be paid to this dataset. Additional investigations are to be performed in order to provide reasons for the decision whether the difference is a consequence of the properties (i.e., types) of the clouds sampled or instrument problems.

Among other statistical methods, the principal component analysis (PCA) is widely used in the atmospheric sciences (see, e.g., Wilks 1995). Having a number of advantages, the PCA technique is used for various applications. One of the most important advantage consists in the fact that the PCA provides the possibility of reproducing almost all the variability of a dataset with reasonably good accuracy while considering only a few principal components. It is also advantageous to use the first principal components in the study if the measured datasets share the main statistical features. It should be noted that the principal components obtained in the course of such study provide by themselves fruitful information. For instance, they can be used in applications when there are needs to model not only cloud mean parameters, but variations of them as well.

The main goal of this paper is to describe a methodology of applying statistical procedures, specifically the PCA technique, to assess data reliability. Another objective is to report on statistical parameters of cirrus 
cloud optical and microphysical characteristics measured during two campaigns of the European INCA experiment (Interhemispheric Differences in Cirrus Properties from Anthropogenic Emissions; Ström et al. 2001). The first campaign, in the Southern Hemisphere (SH), was carried out from Punta Arenas (southern Chile), and the second, in the Northern Hemisphere (NH), from Prestwick (Scotland). Note that the notation NH ("Northern") and SH ("Southern") is used to distinguish the respective campaigns; it is not intended to suggest hemispheric averages. The same instrumentation was operated in both campaigns on board the German DLR Falcon and during the same local season (autumn). In a previous paper (Gayet et al. 2002b), the reliability of the microphysical measurements of four combined and independent techniques had been evaluated from some representative examples. A statistical comparison has also been performed between iceparticle number concentrations measured by the CVI and the FSSP-300 (Seifert et al. 2002).

We first describe the main conditions of measurements and the in situ instruments. Then the principal component analysis technique is discussed. This technique is applied to both the measured angular scattering intensities (i.e., nonnormalized scattering phase functions) and the ice-particle size distributions and allows for a statistical evaluation on measurement reliability for the two datasets in terms of significant components and corresponding eigenvalues.

The PCA technique is used along with the data validation. The validation is provided by intercomparison of parameters derived from different and independent measurements. The statistical relationships between quantities derived from angular scattering intensities and ice-particle distributions, as well as the similarity of the results obtained for the Southern and Northern cases serve as proof of the reliability and reproducibility of the measured cloud properties. Consequently, the two datasets can be used for the comparison between the results from the $\mathrm{SH}$ and $\mathrm{NH}$ campaigns. The differences in cirrus microphysical and optical properties between the two hemispheres, together with interpretation of the results are presented in detail by Gayet et al. (2004).

\section{Instruments and observations}

During the INCA campaigns, the probes were mounted on board the German Falcon research aircraft operated by Deutsches Zentrum für Luft- und Raumfahrt. The INCA dataset includes the $1-\mathrm{Hz}$ measurements performed during 11 flights carried out in the Southern Hemisphere and 10 flights in the Northern Hemisphere. (For additional information on the INCA project and the flight logs we refer to data available online at http://www.pa.op.dlr.de/inca/.)

Three independent techniques were used to measure optical and microphysical properties of cirrus clouds: (i) the polar nephelometer, (ii) the PMS FSSP-300, and (iii) the PMS 2D-C probes. The instruments have already been thoroughly described by Gayet et al. (2002b).

The polar nephelometer (Gayet et al. 1997) measures the angular scattering intensity at the wavelength of 0.8 $\mu \mathrm{m}$ of an ensemble of cloud particles (i.e., water droplets or ice crystals or a mixture of these particles from a few micrometers to about $800 \mu \mathrm{m}$ in diameter). The observations were limited to 32 scattering angles nearuniformly positioned from $15^{\circ}$ to $162^{\circ}$ and restricted to nonpolarized light measurements due to the intrinsic optical setting of the polar nephelometer.

The PMS FSSP-300 optical particle counter basically measures particles from 0.3 to $20 \mu \mathrm{m}$ in diameter (Baumgardner et al. 1992). In the present study the particles larger than $3 \mu \mathrm{m}$ in diameter have been assumed to be ice crystals. Evidence for aspherical small ice particles measured during INCA with typical asymmetry parameter of 0.77 has been highlighted by Gayet et al. (2002b). With regards to the previous work (Gayet et al. 2002b), which considered the calibration proposed by Borrmann et al. (2000), the size calibration of the last channel has been refined. Therefore, the upper size limit of the FSSP-300 in this study is actually $21.8 \mu \mathrm{m}$, which leads to better agreement with the first channel of the 2D-C probe in terms of particle concentration, extinction and ice water content. In this paper we consider the FSSP-300 results only in the size range from 3.0 to $21.8 \mu \mathrm{m}$.

The PMS 2D-C probe provides information on crystal size and shape for the size range $25-800 \mu \mathrm{m}$. The method of data processing used in this study has already been described in detail by Gayet et al. (1996). We recall that the method provides, at a frequency of 1 $\mathrm{Hz}$, the size spectrum distributed over 32 channels (each having a $25-\mu \mathrm{m}$ resolution from a $25-800-\mu \mathrm{m}$ size range) and the usual microphysical parameters: iceparticle concentration, mean particle size, and ice water content (for more details see Gayet et al. 2004).

The inherent shortcomings of the PMS probes and data processing limit the accuracy of derived microphysical parameters as exemplified in Table 1 (Gayet et al. 2004). The rough estimates of random uncertainties (based on published literature) include statistical errors related to sampling statistics, noise errors mainly due to sampling volume determination, and uncertainties from assumptions in the inversion of the PMS probe data (shape, density of particles, airspeed corrections, etc.).

The polar nephelometer is designed to measure the angular scattering intensity of an ensemble of particles. Thus, its sampling volume is much larger (about 200 times) than that of the PMS FSSP-300 and the statistical errors are considerably lower (Table 1). More detailed discussion of the potential problems is given in (Gayet et al. 2004). Considering the sufficiently low level of the polar nephelometer errors, we will use in section 3 the 
TABLE 1. Random uncertainty estimates on microphysical and optical parameters for two particle concentrations: $\mathrm{A}=5 \mathrm{~cm}^{-3}, \mathrm{~B}=$ $0.5 \mathrm{~cm}^{-3}$. Labels (i), (ii), (iii), and (iv) refer to the works of Baumgardner et al. (1992), Gayet et al. (1996), Gayet et al. (2002b), and Gayet et al. (2004), respectively. PMS means FSSP-300 and 2D-C taken altogether.

\begin{tabular}{|c|c|c|c|c|c|c|c|c|}
\hline \multirow{2}{*}{$\begin{array}{c}\text { Parameter } \\
\text { Probe }\end{array}$} & \multicolumn{2}{|c|}{ Particle concentration } & \multicolumn{2}{|c|}{ Particle size } & \multicolumn{2}{|c|}{ Extinction coefficient } & \multirow{2}{*}{$\frac{\text { Phase function }}{\text { Polar neph. (iv) }}$} & \multirow{2}{*}{$\begin{array}{c}\begin{array}{c}\text { Asymmetry } \\
\text { parameter }\end{array} \\
\text { Polar neph. (iii) }\end{array}$} \\
\hline & FSSP-300 (i) & 2D-C (ii) & FSSP-300 (i) & $2 \mathrm{D}-\mathrm{C}$ & PMS (i) & Polar neph. (iii) & & \\
\hline Uncertainty A & $30 \%$ & $50 \%$ & $35 \%$ & $25 \%$ & $60 \%$ & $25 \%$ & $4 \%$ & $4 \%$ \\
\hline Uncertainty B & $75 \%$ & $75 \%$ & $35 \%$ & $25 \%$ & $85 \%$ & $25 \%$ & $4 \%$ & $4 \%$ \\
\hline
\end{tabular}

angular scattering intensity records sampled for $1 \mathrm{~s}$. In section 5 averaging will only be used for synchronization with the PMS probes data.

For both the INCA campaigns, no supercooled water or frozen ice spherical droplets were detected by the polar nephelometer for a temperature below $-33^{\circ} \mathrm{C}$. That is, the corresponding records do not contain data that may be considered as angular scattering intensities of an ensemble of spherical particles. Therefore, the analysis only includes data collected below that temperature. The in-cloud criterion was defined on the basis of the extinction coefficient values derived from the polar nephelometer measurements. The optical and microphysical parameters were related to in-cloud cases when the corresponding extinction coefficient values were larger than a threshold of $0.05 \mathrm{~km}^{-1}$, which roughly corresponds to a concentration of ice particles of $0.1 \mathrm{~cm}^{-3}$ with a diameter of $5 \mu \mathrm{m}$. Accordingly, we deal with a subset of the INCA data and the optically thin cirrus clouds that are not included in the analysis.

In the following, all results are presented separately for the Southern and Northern field experiments. Special attention will be paid to analysis and possible interpretation of the similarity and the difference in characteristics. The following results are obtained on the basis of samples with a total number of 32214 and 21 664 for the Southern and Northern cases, respectively. (This corresponds to around 5476 and $3683 \mathrm{~km}$ of the total length of the in-cloud flights.) One sample means, a data structure that contains optical and microphysical records all together.

\section{Angular scattering intensities from polar nephelometer measurements}

The angular dependence of the irradiance scattered by cloud particles-that is, the single-scattering phase function $\sigma(\theta)$ with $\theta$ representing the scattering anglebelongs to a set of the most important cloud optical characteristics. The phase function and/or its derived parameters, such as the asymmetry parameter, are widely used in applications for climate modeling and remote sensing retrievals. The polar nephelometer provides very important information on the scattering phase function, despite the fact that the measurements are performed in the limited range of angles. In our investigation, we are dealing with an angular scattering intensity (i.e., nonnormalized scattering phase function) expressed in $\left(\mu \mathrm{m}^{-1} \mathrm{sr}^{-1}\right)$ and documented on 32 angular scattering coefficients $\sigma\left(\theta_{i}\right)$, where the parameter $i$ represents the channel number. As we will demonstrate later in this section, the probability density function of the logarithm of cirrus cloud scattered energy values at each scattering angle fit very closely to the normal distribution. Therefore, the statistical study of reliability of the polar nephelometer data has been performed not for the measured values $\sigma\left(\theta_{i}\right)$ but for their logarithm, that is, $1 n \sigma\left(\theta_{i}\right)$.

\section{a. Results from the principal component analysis}

The PCA is known to be designed to generate a new set of uncorrelated parameters, called principal components, representative of the original dataset. Algebraically, principal components could be defined as particular linear combinations of a set of variables. These linear combinations represent the selection of a new coordinate system obtained by rotating the original system of coordinates. The new axes correspond to the directions with maximum variability and provide a simpler description of the covariance structure of the original set of variables (Johnson and Wichern 1998). In many practical implementations, only a few principal components, called significant components, are required to reproduce almost all the system's variability with reasonably good accuracy (see, e.g., Wilks 1995). This is especially true when a limited number of primary physical parameters have a major impact on the measured functions (in this case, angular scattering intensities).

According to the PCA technique, the principal components $\eta_{l}\left(\theta_{i}\right)$, that is, the first eigenvectors of the dataset correlation matrix, allow for the angular scattering intensities (expressed in log scale) to be described with the following expansions (for more details see Jourdan et al. 2003):

$$
\ln \sigma_{j}\left(\theta_{i}\right) \approx\left\langle\ln \sigma\left(\theta_{i}\right)\right\rangle+\sum_{l=1}^{4} C_{j l} \eta_{l}\left(\theta_{i}\right),
$$

where $\langle\ldots\rangle$ denotes averaging over the total dataset, $j$ is the number of the function from the set, and $C_{j, l}=\left(\ln \sigma_{i}\right.$ $-\langle\ln \boldsymbol{\sigma}\rangle)^{\mathrm{T}} \vec{\eta}_{l}$ are the expansion coefficients of $\ln \boldsymbol{\sigma}_{j}\left({ }^{\mathrm{T}}\right.$ denotes a transposed matrix). Each vector (i.e., $\ln \boldsymbol{\sigma}_{j}$ at 
each $j$ ) is expressed with good accuracy by four coefficients $C_{j, l}$. At the same time, the considerably reduced number of parameters $\left(C_{j, l,}, l=1, \ldots, 4\right)$ is much more convenient for further analysis than the vectors $\left[\boldsymbol{\sigma}_{j}\left(\theta_{i}\right)\right.$, $i=1, \ldots, 32]$.

\section{1) ELIMINATION OF NONRELIABLE CHANNELS AND DATA OF DISTINGUISHING CASES}

First of all, the PCA can be used as a tool for impartial elimination of the data corresponding to the nonreliable channels. This relates to channels having had some nonobvious problems (e.g., electronic noise of a rather high level or defective records) during measurements. If this is the case, the first eigenvectors $\eta_{l}\left(\theta_{i}\right)$ graphs have high discontinuities (peaks). Usually, the values of the angular scattering intensities at the neighbor angles are well correlated and the principal components have to be pretty smooth functions. Accordingly, the peaks indicate the nonreliable channels (see the detailed example in Jourdan et al. 2003). As far as it is concerned the INCA dataset, the two channels $\left(102.5^{\circ}\right.$ and $\left.134^{\circ}\right)$ were eliminated on the base of the approach described and the records of the angular scattering intensities at the 30 remaining channels are used in the following.

It is worth noting that the PCA technique can be employed in the "bad data pursuit" wherever the measured parameters are supposed to be reasonably correlated-for example, such parameters as angular or spectral functions of the irradiance that are obtained from remote sensing. Another important point is that the described approach is not able to discover biases, for instance, caused by improper calibrations or wellcorrelated errors in data processing algorithms. On the other hand, the validation is a rather convenient tool to check out if data are biased or not (see section 5).

After eliminating the nonreliable channels, we applied the PCA to the remaining set of angular scattering intensities once more. As expected, the high discontinuities in the principal components disappeared.

It was stated in the introduction that the PCA technique could be employed in the study if the recorded data are statistically homogeneous. The probability distribution functions (PDFs) of the expansion coefficients or their linear combinations as well as the scatterplots $C_{j, n}$ versus $C_{j, m}$ are fruitful in such a study. Usually, if there is a distinguishing subensemble in a dataset, a rigorous investigation of all available parameters has to be performed for the final decision. It was found on the base of such an investigation that in the INCA dataset, the records corresponding to the orographic wave cirrus (OWC) sampled over Punta Arenas on 13 April 2000 and the anvil cirrus (AC) sampled over Prestwick on 12 October 2000 clearly differed from those recorded during other flights. We chose the polar nephelometer data of the OWC case to show an example. In that particular case, the discrepancy is most clearly seen from PDFs of the quantity

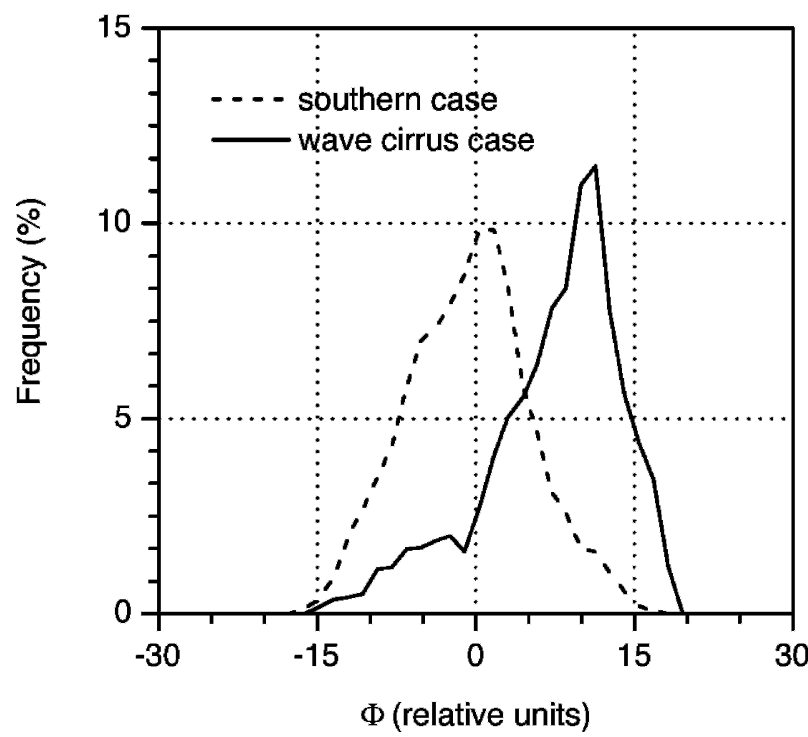

FIG. 1. Probability distribution functions of the parameter $\Phi$ for the Southern case (dashed line) and the wave-cirrus case (black line).

$\Phi_{j}=C_{j, 1}-C_{j, 2}-C_{j, 3}$. Figure 1 shows the normalized to $100 \%$ PDFs of $\Phi$. The black graph corresponds to the OWC data, the dashed curve relates to the Southern data without the wave cirrus case. It is seen that the difference is pronounced and statistically significant. This conclusion is in agreement with the PMS probe data. In the particular OWC and AC cases, the particle number concentration reached very high values (up to $100 \mathrm{~cm}^{-3}$; Gayet et al. 2002c), which were much larger than the "usual" particle concentrations observed in the other cases.

It follows that the sampled orographic wave-cirrus and anvil-cirrus records differ from other INCA data by their optical and microphysical properties; that is, they belong to other statistical ensembles. Accordingly, the full dataset has to be separated into subsets. The same tactics should be applied to each of them. For simplicity's sake, we will demonstrate the results of the data reliability assessment only on the example of the largest subset; that is, we will investigate the INCA data without the two special cases mentioned. (This remark concerns the microphysical data as well.) It will be seen that the remaining ensemble of data is statistically homogeneous and no other subsets have to be eliminated.

\section{2) COMPARING STATISTICAL PARAMETERS}

In a detailed study if data belong to the same statistical ensemble - that is, share main statistical featuresit is convenient to divide data into several subsets and to compare computed characteristics. From a statistical point of view, it is preferable to perform data separation into subsets in an arbitrary and random way. On the contrary, we used the natural subsets of the INCA 


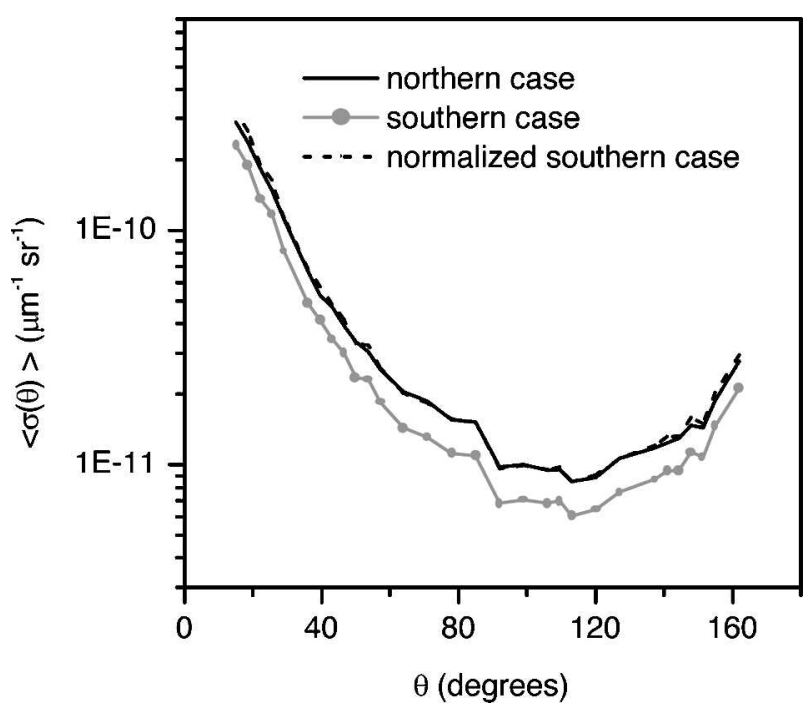

FIG. 2. Average phase functions for the Southern case (gray line with points), the Southern case normalized to the Northern one (dashed line), and the Northern case (black line).

data, that is, the Southern and Northern ones. This allows us to get ideas of distinctions between the data of the two campaigns along with the data reliability assessment.

The average angular scattering intensities calculated for the Southern and Northern datasets are plotted in Fig. 2. (The accuracy of the angular scattering intensity measurements is quite high. Accordingly, the size of the symbols outlines error bars in log presentation.) The results clearly show that the Northern mean values are significantly higher than the Southern ones. This observation is discussed in detail in (Gayet et al. 2004). In addition, some differences are evident in the shapes of the functions by normalizing at $\theta=113^{\circ}$ the Southern average angular scattering intensity to the Northern one (see Fig. 2). It appears that compared to the Northern case, more energy is scattered at the angle range between $15^{\circ}$ and $30^{\circ}$ in the Southern case leading to a slightly larger value of the asymmetry parameter $g$. The mean values of $g$ are 0.770 and 0.768 for the Southern and Northern campaigns, respectively.

After having shown distinctions between the mean values, we are going to discuss characteristics that are similar. The first step was to investigate the linear correlations between the logarithms of the scattered energy values measured at different angles. The results show very high and similar correlation coefficients for both the Southern and Northern cases (greater than 0.95 and 0.97 , respectively). This indicates that the data of the disputable channels were well eliminated and that both sets are rather homogeneous. The variations of the shape of the scattering functions due to the iceparticle shape and/or dimension distinctions can be investigated by employing the PCA technique.

We applied the PCA separately to the Southern and Northern datasets. The results in Figs. $3 \mathrm{a}$ and $3 \mathrm{~b}$ represent the first two principal components or eigenvectors $\eta_{n}\left(\theta_{i}\right)$ of the correlation matrices, where $n$ is the component's number. The results highlight that the Southern $\eta_{n}\left(\theta_{i}\right)_{\mathrm{S}}$ and Northern $\eta_{n}\left(\theta_{i}\right)_{\mathrm{N}}$ eigenvectors are very similar for each component number and furthermore have very close values. For both cases, the values of the first component $\eta_{1}\left(\theta_{i}\right)$ are approximately constant (see Fig. 3a). It should be noted that a strictly constant principal component relates to the case when only the magnitude of the angular scattering intensity varies without any change in the function shape. In turn, this principal component can be associated with the variation of the total cloud particle concentration when the ice-particle shape and the effective diameter remain unchanged. The corresponding eigenvalues of the first components $\delta_{1 \mathrm{~S}}$ and $\delta_{1 \mathrm{~N}}$, normalized as a percentage of the total variance, are of $98.9 \%$ and $99.4 \%$ for the Southern and Northern datasets, respectively (see Table 2). This means that about $99 \%$ of the angular scattering intensity variance is roughly due to fluctuations of the total ice-particle concentration.
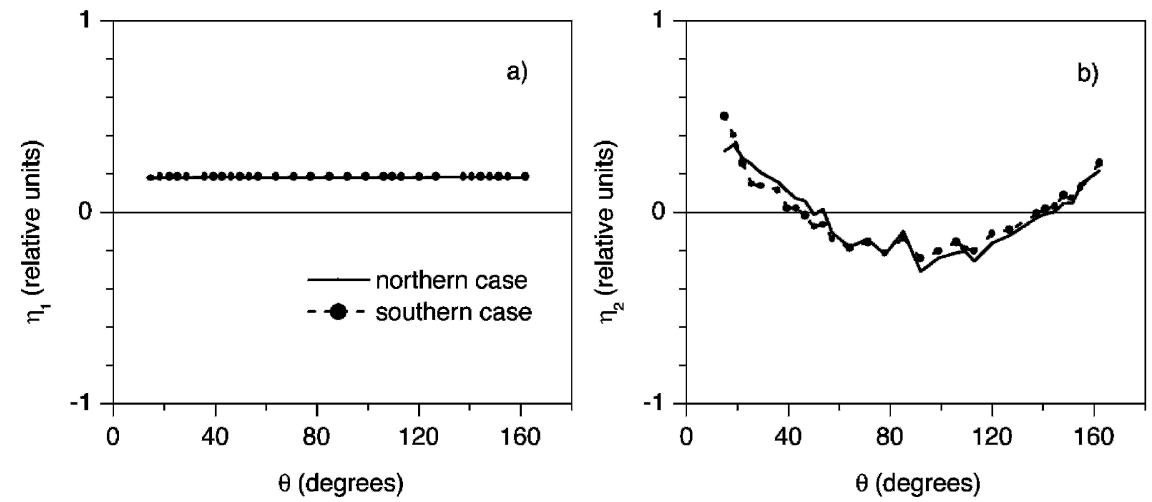

FIG. 3. Principal components of the phase function datasets: (a) first and (b) second. The Southern case is the dashed line with points, the Northern case is the black line. 
TABLE 2. Normalized eigenvalues (in \%) for the first four components calculated for the polar nephelometer measurements. Labels $\mathrm{S}, \mathrm{N}$, and $\mathrm{W}$ correspond to Southern cases, Northern cases, and the whole dataset, respectively.

\begin{tabular}{ccccc}
\hline \hline Component No. & 1 & 2 & 3 & 4 \\
\hline$\delta_{\mathrm{S}}$ & 98.9 & 0.50 & 0.19 & 0.10 \\
$\delta_{\mathrm{N}}$ & 99.4 & 0.28 & 0.12 & 0.04 \\
$\delta_{\mathrm{W}}$ & 99.1 & 0.40 & 0.14 & 0.09 \\
\hline
\end{tabular}

Figure $3 \mathrm{~b}$ displays the second significant component, which is related to the variations of the shape of the angular scattering intensity. The components $\eta_{2}\left(\theta_{i}\right)_{\mathrm{S}}$ and $\eta_{2}\left(\theta_{i}\right)_{\mathrm{N}}$ change their sign twice, namely, around $\theta=$ $45^{\circ}$ and $\theta=145^{\circ}$. Accordingly, they mostly govern the redistribution of the scattered energy from the angle range between $45^{\circ}$ and $145^{\circ}$ to the directions with $\theta$ value lower than $45^{\circ}$ and higher than $145^{\circ}$, and vice versa.

The similarity between the Southern and Northern cases is preserved in the third and fourth components (not shown). Table 2 summarizes the normalized eigenvalues for the second through to the fourth components. The second and the third eigenvalues are $0.50 \%$ and $0.28 \%$ (for the Southern and Northern cases) and $0.19 \%$ and $0.12 \%$, respectively. The second components represent about $46 \%$ of the residual variance (i.e., the variance that remains after considering the first component; Kendall 1980). The second, third, and fourth components taken together represent about $72 \%$ of the residual variance for the two cases.

It should be noted that the principal components are always very sensitive to features of the object investigated. Therefore, the results presented above undoubtedly provide evidence that both angular scattering intensity datasets have the same main statistical properties. This serves as an additional reason to prove the reliability of the polar nephelometer's data. Moreover, this gives grounds to treat both cases mixed together and thus to compute a common set of principal components, that is, a common coordinate system. This coordinate system provides the basis for a more detailed statistical analysis of the data. Therefore, the PCA was applied to the combined dataset. As expected, its results give significant components, which are close to those presented in Fig. 3 (the corresponding normalized eigenvalues are reported in Table 2).

The outlined above data can be used in applications-for instance, to model radiative transfer. Models of the cirrus cloud phase function can be compared against the vectors $\langle\ln \boldsymbol{\sigma}\rangle$ plotted in Fig. 2. Also, the principal components $\eta_{l}\left(\theta_{i}\right)$ along with $\langle\ln \boldsymbol{\sigma}\rangle$ provide an easy way to model variations of the phase function. For this purpose it is enough to use Eq. (1) with $l=1, \ldots$, 2 and vary the coefficient $C_{j, 2}$ within a reasonable range. The advantage of such way is that the variations will be modeled in agreement with the experimental data.

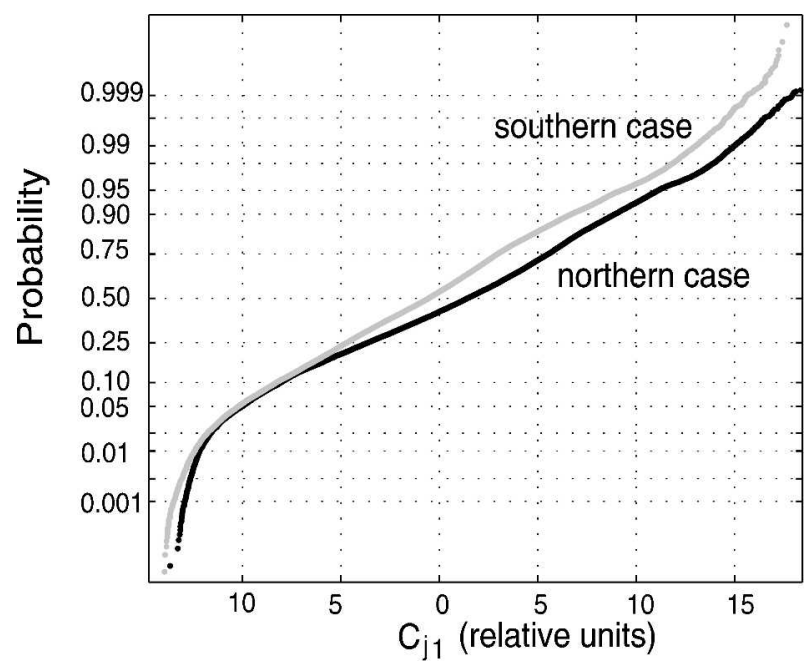

FIG. 4. Cumulative distribution functions of the coefficients $C_{j, 1}$. The Southern case has gray points, the Northern case has black points.

\section{b. Interpretation of the results}

The similarity of the Southern and Northern principal components does not mean that both datasets are totally identical from a statistical point of view, but rather that the main features are the same. There are degrees of freedom that provide a possibility of distinctions, for instance, between the mean values. When the PCA technique is applied to the combined dataset, the difference in the characteristics appears as distinctions between properties of the expansion coefficients $C_{j, l}$. Figure 4 shows a normal probability plot of the coefficients $C_{j, 1}$, computed for the Southern and Northern cases. In such a representation (Chambers et al. 1983), if the data do come from a normal distribution, the plot has to appear linear. The overwhelming majority of points lie close to the straight lines.

The difference in the slope between the two lines reflects the discrepancies between the Southern and Northern average angular scattering intensities. The nonlinear branch at the beginning of each curve is due to the extinction coefficient threshold used to define "in-cloud" observations. Therefore, the angular scattering intensities that correspond to low concentration of ice particles (i.e., the smallest values of $C_{j, 1}$ ) were eliminated along with the clear atmosphere data and offsets. Because the coefficients of the linear correlation between the values of $C_{j, 1}$ and $\ln \sigma_{j}\left(\theta_{i}\right)$ are very high $\left(>0.98\right.$ for all angles $\theta_{i}$ ), the cumulative distribution functions of $\ln \sigma_{j}\left(\theta_{i}\right)$ for each $\theta_{i}$ are strictly close to those represented in Fig. 4 and $\ln \boldsymbol{\sigma}_{j}\left(\theta_{i}\right)$ can be considered as the normal distributions. This property is in agreement with a fundamental result of statistics: the probability density of arbitrary positive quantities is the lognormal probability density (Tarantola 1994).

Two main conclusions follow from the foregoing. 

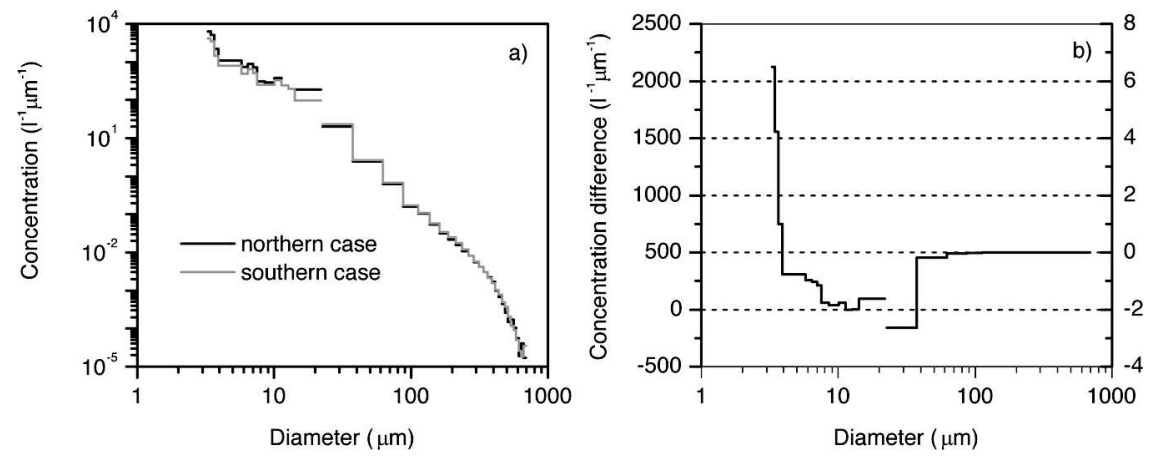

FIG. 5. (a) Average cirrus ice-crystal size distributions. The Southern case is the gray-line histograms, and the Northern case is the black-line histograms. (b) Difference between the Northern and the Southern average cirrus ice crystal size distributions.

First, the probability distribution functions are of the same kind for the Southern and Northern cases. This result is in accordance with the observations of Immler and Schrems (2002) who discuss frequency of occurrence of the optical depth of cirrus clouds during the INCA campaigns. This is another argument for proving the reliability of the polar nephelometer's data. Second, the statement that a variety of cirrus cloud optical characteristics belong to the lognormal distribution can be used in climate and remote sensing applications as well as in inverse problem constraints.

To avoid misunderstanding, the following has to be pointed out. Cirrus cloud single-scattering characteristics do not vary independently, and there is a high correlation between them. However, each parameter by itself can be described by the lognormal law. Another point is that the variety of cirrus cloud types is large. None of parameters can be characterized by a unimodal distribution when experimental data were recorded in several types of clouds or when ice clouds were formed under considerably different conditions.

\section{Particle size distributions from the PMS probes}

The average cirrus ice-crystal size distributions are shown in Fig. 5a for both the Southern and Northern cases. Figure $5 \mathrm{~b}$ illustrates the difference between the distributions (the Northern values minus the Southern ones). The right part of the histograms on Fig. 5a (from 25- to $800-\mu \mathrm{m}$ particle size) displays the data measured by the PMS 2D-C probe. The distinction is given in the corresponding size range of Fig. $5 \mathrm{~b}$ (note the right $y$ axis). The two distributions are very close with the number concentration a little higher for the Southern case. The left part of the histograms of Fig. 5a represents the size distributions measured by the PMS FSSP300 probe. The concentration of relatively small particles is significantly higher for the Northern case (see the left histogram of Fig. 5b). In the size range up to $7.5 \mu \mathrm{m}$, the difference is $2-5$ times higher than the width of a $95 \%$ confidence interval for the estimated average values. The features of the ice crystal size distributions are in full agreement with the properties of the mean angular scattering intensities shown in Fig. 2. It is seen that the preponderance of the Northern angular scattering intensity comes from the dominance of the concentration of the relatively small particles.

Cloud particle size-distributions are derived using the PMS FSSP-300 and PMS 2D-C probes from the numbers of particles counted on each size bin and from the corresponding volume of air sampled. The sampling period $\Delta t$ of $1 \mathrm{~s}$ was used for the optical and microphysical data processing in the INCA project database. Particle counting with an airborne spectrometer is a stochastic Poisson process (see, among others, Pawlowska et al. 1997; Snyder and Miller 1991). Therefore, the relative standard deviation (RSD) increases when the counted number decreases and it is inversely proportional to the square root of the counted number.

With regards to cirrus clouds, it is difficult to ensure a local statistically significant number of counts in every sample. Particle concentration is spatially variable and relatively low, especially the concentration of large particles measured by the PMS 2D-C probe. The sampling volume of the PMS FSSP-300 probe is limited in order to ensure single particle counting. From a statistical significance viewpoint, polar nephelometer data are more reliable because this instrument is designed to measure the angular scattering intensity of an ensemble of particles. Thus, polar nephelometer sampling volume is about 200 times larger than PMS FSSP-300. Inherent random uncertainties including statistical errors related to probe sampling statistics have already been thoroughly discussed with details by Gayet et al. (2002b). Obviously, these random uncertainties become very important for low particle number concentrations. These errors crucially affect results when the objective is, for instance, to study variations of particle size distributions. They could be reduced considerably by averaging over longer periods-that is, by applying linear smoothing with a rectangular window of a fixed sampling period, which corresponds to a low-pass filter. 

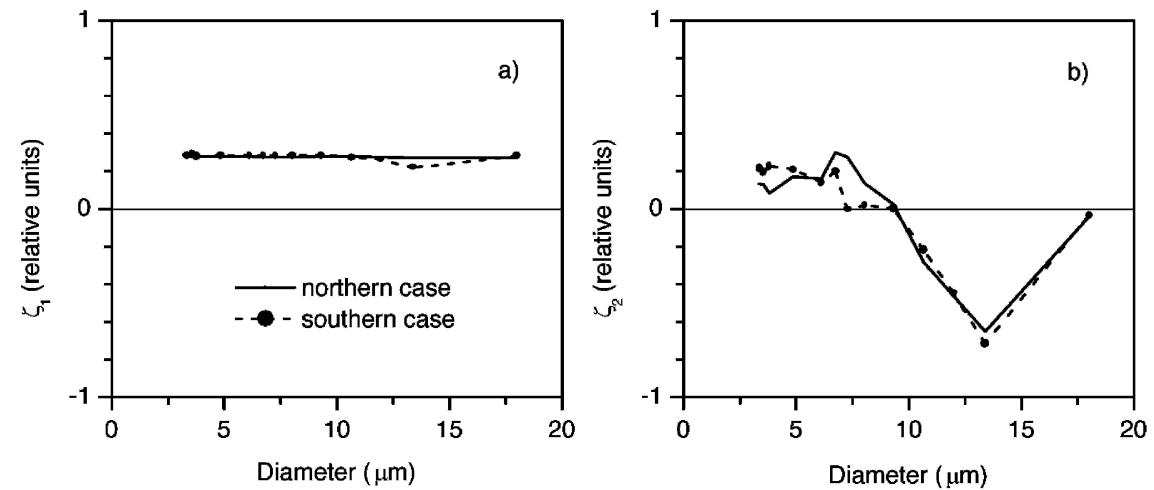

FIG. 6. Principal components of PMS FSSP-300 datasets: (a) first and (b) second. The Southern case is the dashed line with points, and the Northern case is the black line.

Accordingly, such filtering leads to the loss of information on variations at the small scale.

Aiming to apply the PCA technique, we chose a compromise approach. Sampling periods were enlarged for the cases of low concentration and they remain at $1 \mathrm{~s}$ when the RSD is relevant. It was done according to the following algorithm.

It is preassigned the maximal value $\Delta t_{\max }=150 \mathrm{~s}$ of the sampling period and a RSD threshold of $5 \%$. Starting from the first data sample of each flight, the parameter $\Delta t$ is enlarged so long as the RSD is higher than the threshold or $\Delta t$ is lower than $\Delta t_{\text {max }}$. Depending on the particle concentration, the new sampling period $\Delta t_{1}$ obtained can have a value from $1 \mathrm{~s}$ to $\Delta t_{\max }$. The new sample is computed by averaging data belonging to the obtained interval $\Delta t_{1}$. The parameter $\Delta t_{2}$ is computed in the same way starting from the first sample following those that were considered at $\Delta t_{1}$, and so on. As a result, one obtains the set of unequal sampling periods $\Delta t_{k}$ and the new series of corresponding samples. A special procedure should be used at regions where functions jump. Averaging has to be prevented from taking data from intervals with low and high concentrations together. To synchronize filtered data from different probes, the described algorithm was applied to the PMS FSSP-300 records only. Polar nephelometer and PMS 2D-C data were averaged on the base of the series of $\Delta t_{k}$, which came from filtering of the PMS FSSP-300 records. In the following, we deal with the filtered data only.

Because the histogram presentation is not sensitive enough to evidence minor differences between the two datasets, we turn to the PCA technique for a better statistical description of the results. Figure 6 displays two first principal components $\zeta_{n}\left(D_{i}\right)$ of PMS FSSP-300 data. The results show that the Southern and Northern cases present very similar properties. The first components $\zeta_{1}\left(D_{i}\right)_{\mathrm{S}}$ and $\zeta_{1}\left(D_{i}\right)_{\mathrm{N}}$ (Fig. 6a) mostly correspond to variations of the total particle concentration. The second vectors $\zeta_{1}\left(D_{i}\right)_{\mathrm{S}}$ and $\zeta_{2}\left(D_{i}\right)_{\mathrm{N}}$ (Fig. 6b) govern changes of the effective diameter. Table 3 summarizes the normalized eigenvalues for the first and second components. They are $86.0 \%$ and $94.5 \%$ (for the Southern and Northern cases), and $5.7 \%$ and $1.2 \%$, respectively. Accordingly, variations of the particle size distribution shape are more likely for cirrus clouds sampled in the Punta Arenas area. Taken together, the first two significant components represent about $92 \%$ and $96 \%$ of the total variance for the Southern and Northern cases, respectively.

Figure 7 represents the first two principal components $\chi_{n}\left(D_{i}\right)$ of PMS 2D-C data, which again highlight that the results are very similar comparing the Southern and the Northern cases. The first component displayed on Fig. 7a $\left[\chi_{1}\left(D_{i}\right)\right]$ reveals that the variations of concentration of particles with diameters from 200 to 400 $\mu \mathrm{m}$ dominate the feature. Compared to previous results the eigenvalues related to the PMS 2D-C are quite different (see Table 4). They are 30.8\% and 35.0\% (for the Southern and Northern cases) and $20.4 \%$ and $15.7 \%$, respectively. Taken together, the first two significant components represent only about $65 \%$ and $63 \%$ of the total variance for the Southern and Northern cases, respectively. This result arises from the relatively low linear correlation between values of the size distributions of large crystals.

As mentioned above, PCA results are sensitive to minor variations of an observed dataset. Nevertheless, the foregoing clearly shows that the significant components of the size distributions as well as the normalized eigenvalues are close for the Southern and Northern cases. These results serve as an additional reason to prove the reliability of the data recorded by the PMS

TABLE 3. Normalized eigenvalues ( $\%$ ) for the first two components calculated for the PMS FSSP-300 measurements. Labels S and $\mathrm{N}$ correspond to the Southern and Northern cases, respectively.

\begin{tabular}{ccc}
\hline \hline Component No. & 1 & 2 \\
\hline$\delta_{\mathrm{S}}$ & 86.0 & 5.7 \\
$\delta_{\mathrm{N}}$ & 94.5 & 1.2 \\
\hline
\end{tabular}



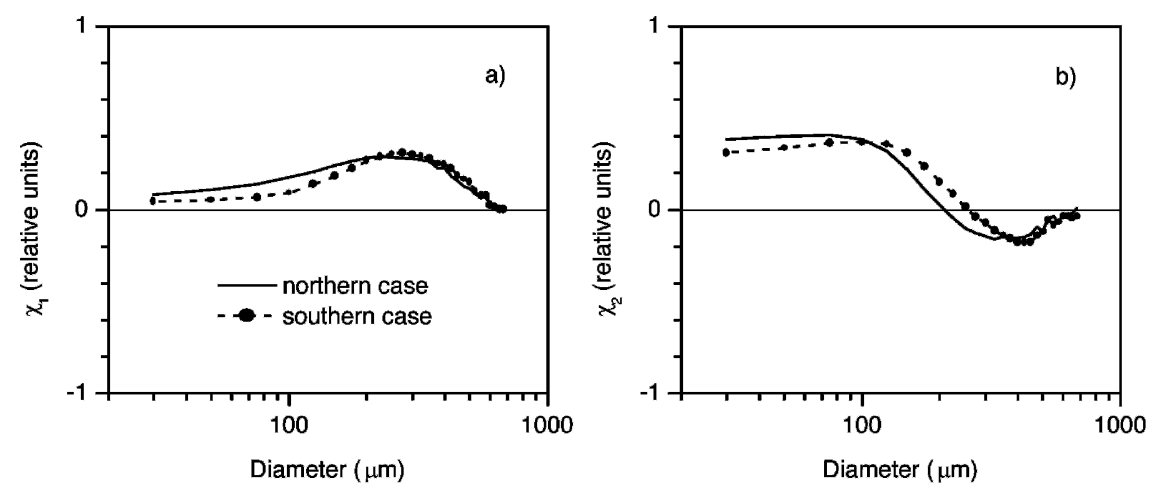

FIG. 7. Principal components of PMS 2D-C datasets: (a) first and (b) second. The Southern case is the dashed line with points; the Northern case is the black line.

FSSP-300 and PMS 2D-C probes during the INCA field campaigns. Also, it should be noted that the average size distributions and the principal components outlined above can be used in applications in the same manner as the data reported in section $3 \mathrm{a}(2)$.

\section{Intercomparison of parameters derived from the polar nephelometer and the PMS probes}

In sections 3 and 4 , it was shown that the Southern and Northern INCA datasets share the main statistical features and, consequently, the optical and microphysical records are not self-contradictory. As mentioned above, the PCA technique is not able to discover biases, for example, caused by improper calibrations or wellcorrelated errors in data processing algorithms. This is why it is highly desirable to employ the PCA along with procedures of validation - that is, comparing estimations of the same parameters derived from data recorded by different probes.

In the following, the intercomparison of the extinction coefficient estimations is presented. The processing method of the INCA data from FSSP-300 and 2D-C probes and the uncertainties on the derived microphysical parameters have been described in detail in Gayet et al. (2002b, 2004). The derivation of the extinction coefficient and the asymmetry parameter from angular scattering intensity measurements documented in a limited range of angles can be found in Gayet et al. (2002a). (Random uncertainty estimates on the extinction coefficient are outlined in Table 1.) Those two techniques can be considered as quite independent. They use different optical characteristics of particles, and the polar nephelometer measures angular scattering intensities of an ensemble of particles, whereas the PMS probe results are derived from single event counting.

Figure 8 displays in $\log -\log$ scale the extinction coefficient inferred from the polar nephelometer as a function of the extinction derived from both the FSSP300 and the $2 \mathrm{D}-\mathrm{C}$ probes. The $\log -\log$ scale is used according to the results of section $3 \mathrm{~b}$ and in order to avoid dominance of the cases with higher extinction values. Scatterplots from the Southern case (Fig. 8a) and the Northern case (Fig. 8b) are represented. We recall that filtered sampled data are used in this comparison. The examination of Fig. 8 confirms that the extinction coefficients derived from independent techniques are in due agreement throughout the large range of the magnitude. In some instances the discrepancy is rather high. Enlarging of sampling periods did not allow us to completely overcome the inherent shortcomings of the probes. The two datasets are characterized by relationships having very close values of the slope parameters. The correlation coefficients are of 0.89 with the $99 \%$ confidence interval from 0.876 to 0.903 for the Southern case, and of 0.82 with the $99 \%$ confidence interval from 0.796 to 0.841 for the Northern case. This result gives evidence for the reliability of the measurements of ice particles by both PMS and polar nephelometer probes and on our method of data processing.

\section{Conclusions}

This paper describes the methodology of applying statistical procedures and, in particular, the principal component analysis technique to assess cirrus cloud data reliability. The approach is demonstrated by an example of a statistical reliability study of optical and microphysical properties measured during two campaigns of the European INCA experiment. The PCA technique was applied to both the measured angular

TABLE 4. Normalized eigenvalues (in \%) for the first two components calculated for the PMS 2D-C measurements. Labels S and $\mathrm{N}$ correspond to the Southern and Northern cases, respectively.

\begin{tabular}{ccc}
\hline \hline Component No. & 1 & 2 \\
\hline$\delta_{\mathrm{S}}$ & 30.8 & 20.4 \\
$\delta_{\mathrm{N}}$ & 35.0 & 15.7 \\
\hline
\end{tabular}



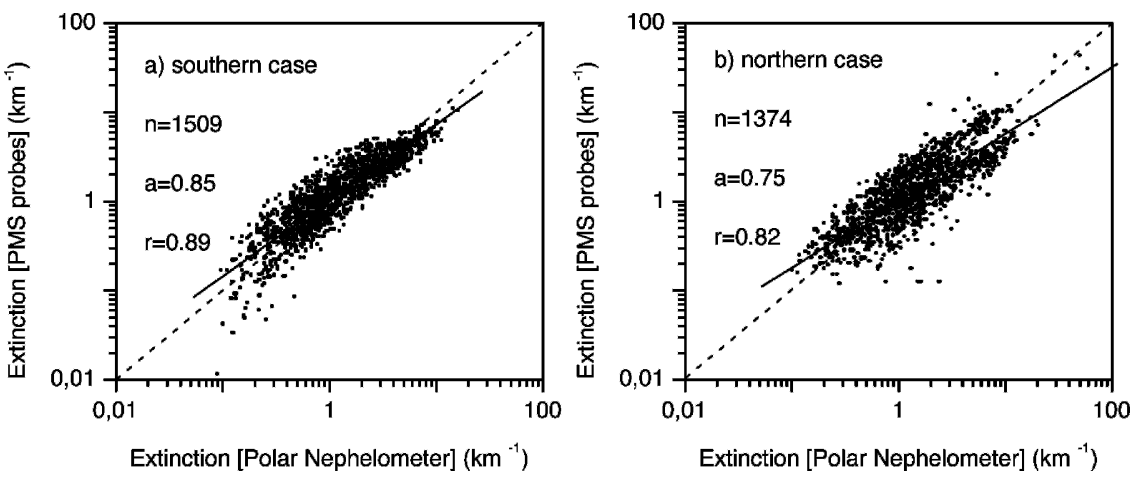

FIG. 8. Extinction coefficient inferred from the scattering phase function vs the extinction calculated from the PMS FSSP and 2D-C probes: (a) Northern case and (b) Southern case. The symbols $n, a$, and $r$ refer to the number of data points, the slope of the best-fit curve equation (solid line), and the correlation coefficient, respectively. The following relationship is assumed to fit the data: $\log (y)=a \bullet \log (x)+b$.

scattering intensities and the ice-particle size distributions.

It is shown that the approach allows for impartial elimination of data not obviously contaminated-for instance, by electronic noise and/or defective recordsthat is, the nonreliable channels of instruments.

The PCA technique can serve in a study if the dataset is statistically homogeneous and provide the possibility of eliminating records corresponding to distinguishing statistical ensembles. It follows from the analysis that the sampled orographic wave-cirrus and anvil-cirrus clouds differ from other INCA data by their optical and microphysical properties.

The PCA results, expressed in terms of significant components and corresponding eigenvalues, show that the Southern and Northern datasets can be considered as statistically representative of the cirrus sampled. Furthermore, the frequency distributions of the cirrus cloud microphysical and optical properties can be considered as arbitrary positive quantities, which are lognormally distributed.

The validation of the measurements is provided by intercomparison of parameters derived from different and independent techniques. Because the instruments have very different sampling volumes, random uncertainties related to sampling statistics could be reduced considerably by adjusting the sampling periods as a function of the particle number concentration. The statistical relationships between quantities derived from angular scattering intensities and ice-particle distributions as well as the similarity of the results obtained for the Southern and Northern cases serve as proof of the reliability of the measured cloud properties. Consequently the two datasets can be used for the comparison between the results from the $\mathrm{SH}$ and $\mathrm{NH}$ campaigns. The differences in cirrus microphysical and optical properties between the two hemispheres are presented and discussed in detail in (Gayet et al. 2004).

The described cirrus cloud characteristics-namely, the average angular scattering intensities, the average size distributions, and the principal components - can be used in applications; for instance, to model radiative transfer. They provide the possibility to simulate variations of parameters in agreement with the experimental data.

Acknowledgments. This work was partially funded by the European Union Contract EVK2-CT-1999-00039, by DLR within the PAZI project, and grants from the CNRS/PATOM. Thanks are due to our INCA colleagues for their helpful contribution to the experiments. We are very grateful to the members of the DLR who operated the Falcon aircraft during the two experiments. We acknowledge all the people that supported us at the Punta Arenas and Prestwick airports both before and during the campaigns. We also thank J-F Fournol and C. Gourbeyre (LaMP) for their technical assistance.

\section{REFERENCES}

American Meteorological Society, 2002: Session 2: Data Reliability and Usability. Preprints, 13th Conf. on Applied Climatology, Portland, OR, Amer. Meteor. Soc., 2.1-2.16.

Baumgardner, D., J. E. Dye, R. G. Knollenberg, and B. W. Gandrud, 1992: Interpretation of measurements made by the FSSP-300X during the Airborne Arctic Stratospheric Expedition. J. Geophys. Res., 97, 8035-8046.

Borrmann, S., B. Luo, and M. Mishchenko, 2000: Application of the T-Matrix method to the measurement of aspherical (ellipsoidal) particles with Forward Scattering Optical counters. J. Aerosol Sci., 31, 789-799.

Chambers, J., W. Cleveland, B. Kleiner, and P. Tukey, 1983: Graphical Methods for Data Analysis. Wadsworth Publishing, $395 \mathrm{pp}$.

Gayet, J.-F., G. Febvre, G. Brogniez, H. Chepfer, W. Renger, and P. Wendling, 1996: Microphysical and optical properties of cirrus and contrails: Cloud field study on 13 October 1989. J. Atmos. Sci., 53, 126-138.

—, O. Crépel, J.-F. Fournol, and S. Oshchepkov, 1997: A new airborne Polar Nephelometer for the measurements of opti- 
cal and microphysical cloud properties. Part I: Theoretical design. Ann. Geophys., 15, 451-459.

- S. Asano, A. Yamazaki, A. Uchiyama, A. Sinyuk, O. Jourdan, and F. Auriol, 2002a: Two case studies of winter continental-type water and mixed-phase stratocumuli over the sea 1. Microphysical and optical properties. J. Geophys. Res., 107, 4569, doi:10.1029/2001JD001106.

— microphysical and optical properties of cirrus clouds with four different in situ probes: Evidence of small ice crystals. Geophys. Res. Lett., 29, 2230, doi:10.1029/2001GL014342.

— , F. Immler, F. Auriol, A. Minikin, A. Petzold, J. Ovarlez, and J. Ström, 2002c: Microphysical and optical properties of a wave-cirrus cloud sampled during the INCA experiment. Preprints, 11th Conf. on Cloud Physics, Ogden, UT, Amer. Meteor. Soc., CD-ROM, P1.2.

—_, and Coauthors, 2004: Cirrus cloud microphysical and optical properties at southern and northern mid-latitudes during the INCA experiment. J. Geophys. Res., in press.

Gonzalez, A., P. Wendling, B. Mayer, J.-F. Gayet, and T. Rother, 2002: Remote sensing of cirrus cloud properties in the presence of lower clouds: An ATSR-2 case study during the Interhemispheric differences in cirrus properties from anthropogenic emissions (INCA) experiment. J. Geophys. Res., 107, 4693, doi:10.1029/2002JD002535.

Heymsfield, A. J., A. Bansemer, P. R. Field, S. L. Durden, J. L. Stith, J. E. Dye, W. Hall, and C. A. Grainger, 2002: Observations and parameterizations of particle size distributions in deep tropical cirrus and stratiform precipitating clouds: Results from in situ observations in TRMM field campaigns. $J$. Atmos. Sci., 59, 3457-3491.

Immler, F., and O. Schrems, 2002: LIDAR measurements of cirrus clouds in the northern and southern midlatitudes during INCA $\left(55^{\circ} \mathrm{N}, 53^{\circ} \mathrm{S}\right)$ : A comparative study. Geophys. Res. Let., 29, 1809, doi:10.1029/2002GL015077.

Johnson, R. A., and D. J. Wichern, 1998: Applied Multivariate Statistical Analysis. 4th ed. Prentice Hall, 816 pp.

Jourdan, O., S. Oshchepkov, J.-F. Gayet, V. Shcherbakov, and H. Isaka, 2003: Statistical analysis of cloud light scattering and microphysical properties obtained from airborne measurements. J. Geophys. Res., 108, 4155, doi:10.1029/2002JD002723.

Kendall, M. G., 1980: Multivariate Analysis. 2d ed. Charles Griffin, $210 \mathrm{pp}$.

Lawson, P., A. J. Heymsfield, S. M. Aulenbach, and T. L. Jensen, 1998: Shapes, sizes and light scattering properties of ice crystals in cirrus and a persistent contrail during SUCCESS. Geophys. Res. Lett., 25, 1331-1334.

McNeil, P., 1990: Research Methods. 2d ed. Routledge, 320 pp.

Menne, M. J., and C. E. Duchon, 2001: A method for monthly detection of inhomogeneities and errors in daily and minimum temperatures maximum. J. Atmos. Oceanic Technol., 18, 1136-1149.

Pawlowska, H., J.-L. Brenguier, and G. Salut, 1997: Optimal nonlinear estimation for cloud particle measurements. J. Atmos. Oceanic Technol., 14, 88-104.

Platt, C. M. R., J. D. Spinhirne, and W. D. Hart, 1989: Optical and microphysical properties of a cold cirrus cloud: evidence for regions of small ice particles. J. Geophys. Res., 94 (D8), 11 151-11 164

Randall, D. A., B. Albrecht, S. Cox, D. Johnson, P. Minnis, W. Rossow, and D. Starr, 1995: On FIRE at Ten. Advances in Geophysics, Vol. 38, Academic Press, 37-177.

Raschke, E., J. Schmetz, J. Heintzenberg, R. Kandel, and R. Saunders, 1990: The International Cirrus Experiment (ICE): A joint European effort. Eur. Space Agency J., 14, 193-199.

Sauvage, L., H. Chepfer, V. Trouillet, P. H. Flamant, G. Brogniez, J. Pelon, and F. Albers, 1999: Remote sensing of cirrus radiative properties during EUCREX'94. Case study of 17 April 1994. Part I: Observations. Mon. Wea. Rev., 127, 504 519.

Schröder, F., and Coauthors, 2000: On the transition of contrails into cirrus clouds. J. Atmos. Sci., 57, 464-480.

Seifert, M., J. Ström, R. Krejci, A. Minikin, A. Petzold, J.-F. Gayet, U. Schumann, and J. Ovarlez, 2002: In situ observations of aerosol particles remaining from evaporated cirrus crystals: comparing clean and polluted cases. Atmos. Chem. Phys. Discuss., 2, 1599-1633.

Snyder, D. L., and M. J. Miller, 1991: Random Point Processes in Time and Space. Springer-Verlag, $480 \mathrm{pp}$.

Starr, D. O'C., and D. P. Wylie, 1990: The 27-28 October 1986 FIRE cirrus case study: Meteorology and clouds. Mon. Wea. Rev., 118, 2259-2287.

Strapp, J. W., F. Albers, A. Reuter, A. V. Korolev, U. Maixner, E. Rashke, and Z. Vukovic, 2001: Laboratory measurements of the response of a PMS OAP-2DC. J. Atmos. Oceanic Technol., 18, 1150-1170.

Ström, J., and Coauthors, 2001: Aerosol and cirrus measurements at midlatitudes on the Southern hemisphere: An overview based on the first INCA experiment. Air Pollut. Rep. 74, Rep. EUR 19, 428. EN, European Commission, Brussels, 314 $\mathrm{pp}$

Tarantola, A., 1994: Inverse Problem Theory: Model for Data Fitting and Model Parameter Estimation. 1st ed. Elsevier Science, $601 \mathrm{pp}$.

Wilks, D. S., 1995: Statistical Methods in the Atmospheric Sciences. International Geophysics Series, Vol. 59, Academic Press, $464 \mathrm{pp}$. 